\title{
Utilização medicamentosa por idosos de uma Unidade Básica de Saúde da Família de Fortaleza - CE
}

\author{
Use of medication for seniors of a basic unit of health of the family of Fortaleza - CE \\ El uso de medicación para las personas mayor de una unidad básica de salud \\ de la familia de Fortaleza - CE
}

\author{
Francisca de Fátima Vasconcelos ${ }^{1}$, Janaína Fonseca Victor ${ }^{2}$, \\ Thereza Maria Magalhães Moreira ${ }^{3}$, Thelma Leite de Araújo ${ }^{4}$
}

\begin{abstract}
RESUMO: Buscou-se investigar a utilização de medicamentos entre idosos pertencentes a um grupo de uma Unidade Básica de Saúde da Família da cidade de Fortaleza-Ce. O estudo descritivo teve amostra intencional, com critério de inclusão na informação do uso medicamentoso diário e contínuo por 52 de 125 idosos. Os dados revelaram que 63,4\% usam medicamentos para hipertensão arterial, $23 \%$ para diabetes mellitus e $13,6 \%$ para ambas as doenças. Foi detectado que $28,8 \%$ utilizam subdosagem, $76,9 \%$ tomam de $1-4$ comprimidos/dia, ainda que $23 \%$, desconheçam o motivo para tal e $44,2 \%$ refiram efeitos colaterais. Do total, $84,6 \%$ não têm ajuda familiar para tomar a medicação, $11,5 \%$ moram sozinhos, $63,4 \%$ não são alfabetizados, mas todos descrevem o formato e cor dos seus comprimidos, utilizando locais fáceis para guarda da medicação. Portanto, fazem-se necessárias pesquisas que visem facilitar a compreensão e utilização medicamentosa contínua por idosos.
\end{abstract}

Descritores: Idoso; Uso de medicamentos; Enfermagem

\begin{abstract}
It was looked for to investigate the use of medicines among senior belonging to a group of a Basic Unit of Health of the Family in Fortaleza-Ce. The descriptive study had intentional sample, with inclusion criterion in the information of the use daily and continuous of the medication for 52 of 125 senior. The data revealed that $63,4 \%$ use medicines for arterial hypertension, $23 \%$ for diabetes mellitus and $13,6 \%$ for both diseases. It was detected that $28,8 \%$ use less dose, $76,9 \%$ take of $1-4$ tablets/day, although $23 \%$ ignore the reason for such and $44,2 \%$ refer collateral effects. Of the total, $84,6 \%$ don't have family help to take the medication, $11,5 \%$ live alone, $63,4 \%$ are not alphabetized, but $100 \%$ describe the format and color of your tablets, using easy places for guard of the medication. Therefore, they are made necessary researches that seek to facilitate the understanding and use continuous of medication for senior.
\end{abstract}

Keywords: Aged; Drug utilization; Nursing

RESUMÉN: Se buscaba investigar el uso de medicinas entre la mayor pertenencia a un grupo de una Unidad Básica de Salud de la Familia del Fortaleza-Ce. El estudio descriptivo tenía la muestra intencional, con el criterio de la inclusión en la información del uso diariamente y continuo de la medicación para 52 de 125 mayor. Los datos revelaron que 63,4\% usan las medicinas para la hipertensión arterial, $23 \%$ para lo diabetes mellitus y $13,6 \%$ para ambas enfermedades. Fue descubierto que $28,8 \%$ usan menos dosis, $76,9 \%$ toman de $1-4$ lápidas/día, aunque $23 \%$ ignoran la razón para tal y $44,2 \%$ refieren los efectos colaterales. Del total, $84,6 \%$ no tienen la ayuda familiar para tomar la medicación, $11,5 \%$ viven exclusivamente, no se alfabetizan $63,4 \%$, pero $100 \%$ describen el formato y color de sus lápidas, mientras usando los lugares fáciles para guardia de la medicación. Por consiguiente, son necesarias investigaciones para facilitar la comprensión y uso continuo de medicación para la persona mayor.

Descriptores: Anciano; Utilización de medicamentos; Enfermería

\section{INTRODUÇÃO}

No Brasil, dados do Instituto Brasileiro de Geografia e Estatística (IBGE) referem que o percentual de pessoas acima de 60 anos corresponde a 8,8\% da população geral. Em termos relativos foi o grupo etário que mais cresceu nos últimos anos, principalmente a partir de 1940. Na região Nordeste, o percentual de pessoas acima de 60 anos é de $8,42 \%$ e no Ceará, $8,86 \%{ }^{(1)}$.

De acordo com a Organização Mundial de Saúde (OMS), a população de idosos crescerá de tal modo, que o Brasil será o sexto do mundo, com cerca de 15

\footnotetext{
Enfermeira do PSF. Mestre em Enfermagem pela UFC E-mail: fatimavas@yahoo.com.br

Especialista em Saúde da Família. Doutoranda em Enfermagem pela UFC.

3 Professora adjunto da UECE.

4 Professora adjunto da UFC.
} 
milhões de pessoas com 60 anos ou mais em 2020. Projeções indicam, ainda, que este segmento poderá chegar a quase $15 \%$ do contingente populacional em 2027, superando o número de crianças e adolescentes com 14 anos ou menos ${ }^{(2)}$.

As mudanças demográficas dos idosos são acompanhadas por mudanças epidemiológicas, especialmente de morbimortalidade. As doenças do aparelho circulatório foram a primeira causa de morte entre os idosos brasileiros, nas últimas décadas: em 1980, 44,7\% , em 1991, $40 \%$ e em 1996, 38.1\% do total de óbitos. Em meio às principais doenças do aparelho circulatório, há um predomínio das doenças cerebrovasculares, seguidas pelas doenças isquêmicas do coração. Tais doenças foram as causas mais freqüentes de internações hospitalares, revertendo-se em gastos para o Sistema Único de Saúde (SUS), em torno de $23 \%$, com a referida população, ocorrendo, também, aumento nas reinternações que, em idosos, são cinco vezes maiores do que na faixa etária de 15 a 59 anos $^{(3)}$.

Em decorrência do aparecimento de doenças crônico-degenerativas e suas complicações, os pacientes com mais de 60 anos, são os principais consumidores da farmacoterapia moderna, sendo que $80 \%$ tomam, diariamente, no mínimo, um medicamento e utilizam mais medicamentos que qualquer outro grupo etário e, quando hospitalizados, recebem entre oito a quinze medicamentos por $\mathrm{dia}^{(4-5)}$.

Embora o uso de medicamentos seja uma questão relevante em todas as faixas etárias, as pesquisas sobre o assunto têm se dedicado, com freqüência, ao paciente idoso, em decorrência das peculiaridades desse grupo etário, pois é comum a ocorrência de subdosagem, superdosagem e não adesão à prescrição médica ${ }^{(4)}$.

Relata-se que mais do que em qualquer outro grupo etário, os medicamentos são indicados para os idosos sem clara correspondência entre a doença e a ação farmacológica. Eles são empregados de forma equivocada, como sucedâneos das mudanças para um estilo de vida mais saudável, a prescrição é impulsionada não somente pela real necessidade da utilização de um fármaco, mas também, por uma imposição cultural que o idoso precisa de medicamentos. Este fato conduz a distorções na produção, regulamentação, prescrição e uso de medicamentos, levando quase um quarto dos idosos a receber, no mínimo, um fármaco impróprio ${ }^{(6)}$.

O uso de medicamentos por idosos, também envolve o entendimento das mudanças estruturais e funcionais dos vários órgãos e sistemas, como déficit de visão, perda de memória e diminuição da destreza manual. Estas mudanças podem afetar a capacidade do idoso em compreender adequadamente as orientações do tratamento medicamentoso, podendo confundir-se e tomar medicamentos de maneira incorreta ${ }^{(7)}$.
É relevante que os profissionais de saúde, no acompanhamento de pacientes idosos em uso de medicação, desenvolvam estratégias que contemplem orientações e informações sobre o diagnóstico e terapia utilizada, levando em consideração as mudanças trazidas pelo processo de envelhecimento.

A terapia medicamentosa deve ser enfatizada, não só no discurso inflexível de seguir corretamente a prescrição, mas na comunicação saudável entre profissional e paciente, abordando o tratamento medicamentoso e as condições de escolaridade, custos, presença de cuidador na família, número de comprimidos por dia, dentre outros. $\mathrm{O}$ enfermeiro como membro da equipe de saúde, deve conhecer o processo de envelhecimento, as doenças associa-das e desenvolver estudos, aprimoramentos dos cuidados e estratégias que contribuam para um melhor cuidado às pessoas idosas, incluindo neste cuidado as intervenções adequadas quanto ao uso de medicamentos ${ }^{(4,8)}$.

Ações que busquem analisar a terapia medicamentosa, no intuito de melhorar a qualidade de vida dos idosos, devem ser estimuladas e desenvolvidas por profissionais de saúde. Diante da magnitude dessa problemática e do desejo de contribuir com novas descobertas para um melhor cuidado ao idoso, o presente trabalho buscou investigar o uso de medicamentos entre idosos participantes de um grupo acompanhado em uma Unidade Básica de Saúde da Família (UBASF) de Fortaleza-Ceará, analisando aspectos socioeconômicos dos idosos, o número de medicamentos consumidos, indicações, efeitos colaterais, locais de guarda da medicação e participação familiar.

\section{MATERIAL E MÉTODOS}

A pesquisa descritiva foi realizada em uma Unidade Básica de Saúde da Família (UBASF), do município de Fortaleza-Ceará. A população integrante foi composta por 125 idosos, participantes de um grupo atendido na UBASF, sendo 114 mulheres e 11 homens. Este grupo foi formado desde 2002 pelas enfermeiras da UBASF, com objetivo de facilitar ações de educação em saúde para essa faixa etária. A amostra foi do tipo intencional e o critério utilizado para inclusão, foi à informação do uso diário e contínuo de, pelo menos, um medicamento. Com esse critério, foram selecionados 52 idosos, representando $41,6 \%$ do total de idosos.

Os dados foram coletados por fonte primária (diretamente com os idosos) e o instrumento de coleta utilizado foi o formulário. A adoção deste instrumento permitiu que o pesquisador estivesse presente e que registrasse as respostas. A coleta dos dados foi realizada de agosto a setembro de 2003.

Foram analisadas as variáveis escolaridade, número de medicamentos tomados por dia e suas características 
(nome, cor, indicação), se os idosos têm ajuda na hora de tomar o medicamento, com quem residem, diagnósticos médicos, efeitos colaterais observados com o uso dos medicamentos e locais de guarda da medicação.

O projeto que possibilitou a construção deste artigo foi aprovado pelo Comitê de Ética em Pesquisa do Complexo Hospitalar da Universidade Federal do Ceará. Foram observados os aspectos éticos, conforme resolução 196/96, que estabelece os critérios para pesquisas envolvendo seres humanos, todos concordaram em participar do estudo, após leitura pelos pesquisadores do consentimento livre e esclarecido, sendo thes assegurado sigilo, e anonimato das falas e exposições na divulgação das informações ${ }^{(9)}$.

Os dados foram tratados em termos percentuais, não sendo atribuído julgamento de valor sobre suas exposições.

Acredita-se que o estudo poderá ser utilizado para aprimoramento do cuidado aos pacientes idosos, o que representa sua beneficência.

\section{RESULTADOS E DISCUSSÃO}

Os dados revelaram que, dos 52 idosos selecionados, $63,4 \%$ usam medicamentos para hipertensão arterial, $23 \%$ para diabetes mellitus e $13,6 \%$ para ambas as doenças.

A hipertensão arterial é, sem dú-vida, a doença mais comum nos idosos, seguida pelo diabetes mellitus. Na primeira, a prevalência pode chegar a $20 \%$, na população acima de $60 \operatorname{anos}^{(10)}$.

A possibilidade de associação das duas doenças é da ordem de $50 \%$, pois apresentam vários aspectos comuns, como: etiopatogenia, fatores de risco e cronicidade, dentre outros. A associação de doenças exige maior atenção por parte do idoso, pois aumenta o número de medicamentos usados diariamente ${ }^{(10-11)}$.

Quando indagados sobre quais medicações tomam diariamente, 55,8\% dos idosos entrevistados referem saber o nome. A tabela a seguir traz o tipo de medicamento utilizado.

Tabela 1 - Fármacos utilizados pelos idosos que sabem informar o nome da medicação de uso contínuo. Fortaleza-Ce, 2003.

\begin{tabular}{lr}
\hline \multicolumn{1}{c}{ Medicamento } & $\%$ \\
\hline Adalat & 3,4 \\
Captopril + Hidroclorotiazida + Clorpropamida & 5,5 \\
Captopril + propanolol & 6,8 \\
Hidroclorotiazida & 31,0 \\
Hidroclorotiazida + captopril & 27,5 \\
Hidroclorotiazida + propanolol & 6,8 \\
Insulina NPH & 13,5 \\
Propanolol & 5,5 \\
\hline Total & 100,0 \\
\hline
\end{tabular}

O quadro a seguir, mostra os principais medicamentos utilizados de acordo com o problema de saúde apresentado:

Quadro 1 - Medicamentos mais utilizados, de acordo com o problema de saúde apresentado. Fortaleza-Ce, 2003.

\begin{tabular}{|c|c|c|}
\hline $\begin{array}{c}\text { HIPERTENSÃO } \\
\text { ARTERIAL }\end{array}$ & $\begin{array}{c}\text { HIPERTENSÃO } \\
\text { ARTERIAL + } \\
\text { DIABETES MELLITUS }\end{array}$ & $\begin{array}{c}\text { DIABETES } \\
\text { MELLITUS }\end{array}$ \\
\hline $\begin{array}{c}\text { Captopril } \\
\text { Hidroclorotiazida } \\
\text { Propanolol } \\
\text { Adalat }\end{array}$ & $\begin{array}{c}\text { Captopril } \\
\text { Hidroclorotiazida } \\
\text { Clorpropramida }\end{array}$ & $\begin{array}{c}\text { Insulina NPH } \\
\text { Insulina regular } \\
\text { Clorpropramida }\end{array}$ \\
\hline
\end{tabular}

A hidroclorotiazida é um diurético tiazídico, que pode ser utilizado isoladamente ou associado a outro antihipertensivo. Pode apresentar reações adversas, como: desconforto epigástrico, naúseas, vômitos, hipotensão, cefaléia, distúrbios hidroeletrolíticos, dentre outros ${ }^{(10)}$. Por se tratar de um diurético, deve ser repassado ao idoso que haverá aumento da eliminação urinária e a importância de seguir o horário prescrito, visto que $11 \%$ dos idosos do estudo que fazem uso dessa medicação, não seguiam a prescrição quanto ao horário, trocando o período da manhã pelo noturno, o que dificultava conciliar sono e repouso, devido à nictúria.

O propranolol é um betabloqueador, que pode ser utilizado, tanto no tratamento da hipertensão arterial, como em uma série de condições, tais como: arritmias cardíacas, angina do peito, podendo também ser usado isoladamente ou em associações, embora seja contra-indicado para asmáticos e diabéticos ${ }^{(10)}$.

O captopril é a medicação mais usada pelos idosos do estudo, principalmente, em associação com outras drogas. É classificada como inibidora da Enzima Conversora da Angiotensina (ECA). Tem indicação formal para hipertensos portadores de diabetes mellitus. Os pacientes em uso de captopril podem apresentar efeitos adversos, como: tosse, prurido, hipotensão, distúrbios gastro-intestinais, dentre outros ${ }^{(10,12)}$.

Um dado curioso, é que, do total, $28,8 \%$ dos idosos afirmam "economizar" o medicamento, isto é, deixam de seguir a prescrição, utilizando subdosagem, por iniciativa própria, fato que acontece, principalmente, no final do mês, quando os comprimidos estão chegando ao fim; importante ressaltar, que embora a medicação seja fornecida pela UBASF, há períodos que o fornecimento é irregular. $O$ medo de não ter a medicação na unidade de saúde e a falta de condições econômicas para comprá-la foi o motivo referido para esta conduta.

Segundo o Manual de Hipertensão Arterial e Diabetes Mellitus do Ministério da Saúde ${ }^{(10)}$, em relação ao 
tratamento medicamentoso dessas doenças, os profissionais envolvidos devem de forma contínua lembrar noções básicas quanto ao horário, relação com alimentos, sono, diurese e mecanismo de ação, como estratégia para garantir a manutenção da prescrição. A principal causa de hipertensão arterial resistente é a descontinuidade da prescrição estabelecida.

Os dados revelam que $76,9 \%$ dos idosos do estudo tomam de 1 a 4 comprimidos por dia e $23 \%$ desconhecem o motivo de fazerem uso diário de medicação, não associando sua utilização à presença da hipertensão e do diabetes mellitus, mas aos sintomas e complicações decorrentes dessas doenças ou aos efeitos colaterais medicamentosos. As falas a seguir ilustram a afirmação.

Eu tomo o remédio para melhorar a urina. Quando eu tomo, faço xixi muito bem (P 1).

O remédio que eu tomo serve para as urinas, para inchaço, para melhorar meu sono (P 2).

Eu tomo esse remédio para melhorar o meu cansaço, minha boca seca, minha urina (P3).

Neste estudo, todos os idosos referem somente usar a medicação com prescrição médica, já o estudo realizado por Anderson et al., com 93 idosos revelou que 30\% destes faziam uso de medicação sem prescrição médi$\mathrm{ca}^{(13)}$. Pode-se inferir que o fato dos idosos do estudo pertencerem a um grupo que trabalha questões educativas possa facilitar essa conduta, somado ao fato da relativa facilidade de acesso ao atendimento médico na UBASF.

O acompanhamento de idosos em uso de medicação contínua deve privilegiar orientação e informação periódica do conjunto dos medicamentos e de seus possíveis efeitos adversos. A literatura recomenda a preferência por monodoses em detrimento das associações em doses fixas, preferência por fármacos de eficácia comprovada pelas evidências científicas, verificação da compreensão da prescrição e das orientações farmacológicas, além da simplificação dos esquemas de administração e atenção aos preços ${ }^{(6)}$.

Efeitos colaterais dos medicamentos foram referidos por $44,2 \%$ dos idosos, que suspendem seu uso por dois ou três dias, ao se sentirem muito incomodados pelo fato. Os principais efeitos relatados foram epigastralgias, tonturas, naúseas, tosse, cefaléia e boca seca.

O uso de medicação em idosos apresenta peculiaridades, pois, com a idade, diminui a massa muscular e a água corporal. O metabolismo hepático, os mecanismos homeostáticos, assim como a capacidade de filtração e de excreção renal podem ficar comprometidos. O que leva à dificuldade de eliminação de metabólitos, acúmulo de substâncias tóxicas no orga- nismo e produção de reações adversas. Pode, inclusive, haver superposição entre essas últimas e os quadros mórbidos pré-existentes ${ }^{(6)}$.

Esses fatos reforçam a imagem negativa dos medicamentos em idosos. Estudo desenvolvido por Teixeira e Lefèvre revelou que o principal problema na manutenção do tratamento medicamentoso é o medo dos efeitos secundários, pois associam esses ao surgimento de uma segunda doença ${ }^{(4)}$. Ainda em relação aos efeitos colaterais, Rozenfeld, afirma que o uso de múltiplos produtos, e/ou o uso de dois ou mais fármacos com a mesma atividade e o treinamento inadequado da equipe de saúde, favorecem o aparecimento dos efeitos adversos e das interações medicamentosas em idosos ${ }^{(6)}$.

Os dados revelaram que $63,4 \%$ dos idosos entrevistados não são alfabetizados, o que dificulta o cumprimento das prescrições médicas sem auxílio. Este fato limita a autonomia do idoso e exige maior cuidado na prescrição e orientação profissional. Para a formulação de tipos específicos de intervenção, como no caso de uso de medicação,os profissionais envolvidos devem compreender que as características socioeconômicas e culturais, geralmente, representadas pelas variáveis: renda, ocupação, escolaridade, classe social são importantes, pois interferem no sucesso da intervenção ${ }^{(11)}$.

Para facilitar o uso de medicação em pacientes não alfabetizados, deve-se separar os medicamentos para uso diário em frascos identificados, se possível, com tampas de cores diferentes, conforme o horário em que deverão ser tomados ${ }^{(14)}$. Essa estratégia não foi referida por nenhum idoso do estudo.

Dos entrevistados, 84,6\% não têm ajuda familiar na hora de tomar a medicação, o que deve ser levado em conta pelos profissionais que os atendem, a orientação dispensada ao idoso deve ser diferenciada quanto à dispensada a um membro da família ou cuidador, em ambas situações há particularidades que precisam ser observadas, como exemplos: grau de instrução, déficit visual e auditivo.

Considera-se imprescindível que o idoso seja estimulado a assumir a responsabilidade pelo tratamento, pois o processo de envelhecimento não o torna incapaz, no entanto, quando ocorre uma participação ativa da família, os resultados na manutenção do tratamento podem ser potencializados ${ }^{(14-15)}$.

Observamos que $11,5 \%$ dos idosos do estudo moram sozinhos, fato que limita a participação da família no cuidado e contribui para o isolamento social. Quanto ao idoso morar só, não significa um problema em si, já que pode ser opção. Entretanto, a Organização Mundial de Saúde (OMS), considera esta condição como uma situação de risco, tendo em vista a possibilidade de perda da autonomia, o risco de solidão e sentimentos de vulnerabilidade ${ }^{(13)}$. 
O idoso necessita de atenção no atendimento de suas necessidades biopsicossociais, de incentivo para desenvolver as atividades comunitárias, de valorização das suas histórias de vida, de interação com a família e de inúmeras outras formas para sua integração social ${ }^{(15)}$.

Com referência ao conhecimento das medicações, $100 \%$ dos idosos descrevem o formato e sabem a cor dos comprimidos que tomam diariamente, mas $44,2 \%$ não sabem referir o nome da medicação da qual fazem uso. Na maioria das vezes, quando interrogados sobre a medicação, iniciam suas falas descrevendo os medicamentos, como exemplificado a seguir:

Ah, eu tomo um branquinho, miudinho (P 4).

Eu tomo um branquinho de manhã e um amarelo de manhã e de noite (P 5).

De manhã, eu tomo um branquinho dos grandes e à noite eu tomo de novo (P 6).

Esse fato pode ser explicado pelo alto percentual de não alfabetizados, mas é um dado preocupante, já que medicações diferentes possuem o mesmo formato e cor, o que pode levar a erros na tomada da medicação. Esse dado abre um leque de questionamentos quanto ao tipo de orientação dispensada pelos profissionais de saúde aos idosos não alfabetizados que utilizam medicação diariamente, não somente na UBASF deste estudo, como também, nas demais unidades de saúde, que tipo de estratégia educativa poderá ser utilizada para auxiliar a tomada correta da medicação?

Quanto ao local da guarda da medicação, os idosos citaram que, para lembrar de tomar a medicação, preferem os seguintes locais, demonstrados no gráfico a seguir.

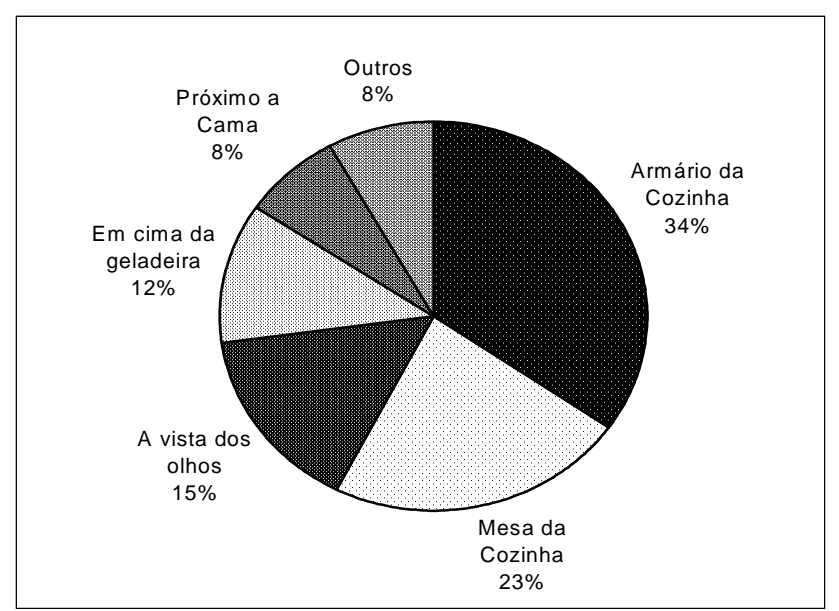

Figura 1 - Local de guarda da medicação escolhido pelos idosos da UBASF, Fortaleza-Ce. Ago.-Set./2003
O local escolhido pelo idoso pode facilitar ou dificultar o uso do medicamento. A grande maioria dos entrevistados $(67 \%)$, escolhe a cozinha como o compartimento da casa para a guarda da medicação, como estratégia de facilitar a tomada correta e de impedir o esquecimento, uma vez que é nesse ambiente que passam a maior parte do tempo, a escolha por esse cômodo da casa pode ser explicado pelo fato que 48 (92\%) dos sujeitos do estudo são do sexo feminino.

Do total dos participantes, $28,8 \%$ referiram que, mesmo deixando em lugar fácil, não se lembram de tomar a medicação diariamente. Sabe-se que o envelhecimento em si, não é condição incapacitante, mas autores como Ventura, Bottino e Diaz referem ser comum o aparecimento de queixas relacionadas a distúrbio de memória no idoso, este pode ser o esquecimento senescente benigno, ou ser decorrentes de processos patológicos ${ }^{(16-17)}$. O profissional de saúde deve estar atento para o fato, que as propriedades cognitivas no paciente idoso podem se encontrar afetadas, resultando em dificuldade para o entendimento ou lembrança correta de seus regimes terapêuticos.

\section{CONCLUSÕES}

O cuidado com a saúde do idoso deve considerar o complexo processo de envelhecer, que ultrapassa barreiras fisiológicas, psicológicas e sociais, pois engloba a realidade econômica, cultural, o contexto familiar e necessidades de ações específicas.

$\mathrm{O}$ estudo revelou que muitos fatores influenciam no correto tratamento medicamentoso, como: capacidade de ler e escrever, desconhecimento sobre os medicamentos, número de comprimidos tomados diariamente, local de guarda do medicamento, contexto familiar, dentre outros.

É oportuno que os profissionais de saúde que prescrevem e orientam a medicação, desenvolvam estratégias que facilitem o cuidado ao idoso. No caso do tratamento medicamentoso, estas estratégias devem adequarse ao contexto social em que o idoso está inserido, além de evitar o uso de linguagem rebus-cada, durante orientações sobre medicação.

Em vista do encontrado, fazem-se necessárias novas pesquisas, que possam caracterizar o idoso brasileiro nos diferentes contextos sociais em que ele se encontra, bem como as possíveis soluções para atender às suas necessidades quanto ao uso contínuo de medicação. 


\section{REFERÊNCIAS}

1. Fundação Instituto Brasileiro de Geografia e Estatística. Censo demográfico 2000: resultados da amostra. [online]. Rio de Janeiro; 2000. Disponível em: <http://www1.ibge.gov.br/home/ estatistica/populacao/cens2000/primeiros_resultados_amostra/ brasil/pdf/tabela_1_1_1.pdf>. (16 out. 2003).

2. Kalache A. Envelhecimento no contexto mundial a perspectiva da Organização mundial da Saúde. In: Anais do Seminário Internacional de Envelhecimento Populacional: uma Agenda para o Final do Século; 1996; Brasília (DF).

3. Costa MFL, Guerra HL, Barreto SM, Guimarães RM. Diagnóstico da situação de saúde da população idosa brasileira: um estudo da mortalidade e das internações públicas. Inf Epidemiol SUS 2000; 9(1):23-41.

4. Teixeira JJV, Lefèvre F. A prescrição medicamentosa sob a ótica do paciente idoso. Rev Saúde Pública 2001; 35(2):207-13.

5. Romano Lieber NS, Teixeira JJV, Farhat FCLG, Ribeiro E, Crozatti MTL, Oliveira GS. Revisão dos estudos de intervenção do farmacêutico no uso de medicamentos por pacientes idosos. Cad Saúde Pública 2002; 18(6):1499-507.

6. Rozenfeld S. Prevalência, fatores associados e mau uso de medicamentos entre os idosos: uma revisão. Cad Saúde Pública 2003; 19(3):717-24.

7. Bison RAP, Furegato ARF, Toledo VP. Perfil e condições de saúde do idoso de um programa de atenção à terceira idade, sob a ótica da enfermagem. Rev RENE 2002; 3(1):9-13.
8. Smelter B, Bare S. Brunnner \& Sttudart: tratado de enfermagem médico-cirurgica. Rio de Janeiro: Guanabara Koogan; 2002.

9. Conselho Nacional de Saúde. Resolução n. 196, de 10 de outubro1996. Dispõe sobre pesquisa envolvendo seres humanos. Bioética 1996; 4(2 Supl):15-25.

10. Ministério da Saúde. Departamento de Atenção Básica. Área Temática de Diabetes e Hipertensão Arterial. Hipertensão Arterial Sistêmica e Diabetes Mellitus: protocolo. Brasília; 2001.

11. Veras RP. Brasil envelhecido e o preconceito social. In:Veras RP, organiza-dor. Terceira idade. Rio de Janeiro: Relume Dumará; 1999.

12. Lima DR. Manual de farmacologia clínica, terapêutica e toxicologia. Rio de Janeiro: Ed. Médica e Científica; 2002.

13. Anderson MIP. Saúde e qualidade de vida na terceira idade. Textos sobre envelhecimento. Rio de Janeiro: UNATI/UERJ; 1998.

14. Ministério da Saúde. Ministério da Previdência e Assistência Social. Idosos: problemas e cuidados básicos. Brasília; 1999.

15. Jacob FilhoW.Promoção da saúde do idoso. São Paulo: Lemos; 1998.

16. Ventura MN, Bottino CMC. Avaliação cognitiva em pacientes idosos. In: Papaléo Neto M. Gerontologia: a velhice e o envelhecimento em visão globalizada. São Paulo: Atheneu; 1996.

17. Diaz RB. Adesão ao tratamento medi-cametoso In: Papaléo Neto M. Geron-tologia: a velhice e o envelhecimento em visão globalizada. São Paulo: Atheneu; 1996.

\section{FORMULÁRIO}
1.Idade
2. Sexo
3. Sabe ler e escrever Sim ( ) Não( )

4. Portador de HAS ( ) DM ( ) HAS + DM ( ) Outros

5. Faz uso de medicamento diariamente, se sim sabe informar o nome?

Sim ( ) Não ( ) Obs

6. Quantos comprimidos o(a) senhor(a) toma diariamente

7. Quando necessita de tomar alguma medicação qual a primeira coisa que o(a) senhor(a) faz?

8. Após tomar o seu remédio o(a) senhor(a) sente alguma alteração no seu corpo?

$\operatorname{Sim}($ ) qual Não ( )

9. O(a) senhor(a) alguma vez, por algum motivo que achou importante, deixou de tomar a medicação prescrita pelo seu médico? Sim ( ) porque Não ( )

10.O(a) senhor(a) sabe para que serve o remédio que toma diariamente?

11. Onde o(a) senhor(a) costuma guardar os seus remédios?

12. O(a) senhor(a) esquece de tomar o seu remédio? Sim ( ) Não ( )

13. O que o(a) senhor(a) costuma fazer para ajudá-lo(a) a não esquecer de tomar a medicação?

14. Com quem o(a) senhor(a) mora? Só ( ) com a família ( )

15. Alguém da sua família ajuda o(a) senhor(a) a tomar sua medicação? Sim ( ) Não ( ) 